\title{
Contamination of Asian herbal drugs : Need for its critical evaluation
}

\author{
K.K. Gupta, G. Prasad", A.K. Chopra ${ }^{1}$ and D.R. Khanna ${ }^{1}$ \\ Department of Botany and Microbiology, Gurukula Kangri University, Haridwar-249404 (Uttarakhand), INDIA \\ ${ }^{1}$ Department of Zoology and Environmental Science, Gurukula Kangri University, Haridwar-249404 (Uttarakhand), INDIA \\ *Corresponding author. E-mail: gpgupta49@yahoo.com
}

\begin{abstract}
Herbal drugs have been used as remedies for the treatment of large number of humans, ailments since ancient times as the traditional medicine system; India has a glorious past, having first documented record of fully developed medical science known as Ayurveda written by several ancient Rishi's. Major groups of indigenous system of medicine are based on herbal drugs. A survey made by WHO indicated that about $80 \%$ of the world population relies on herbal drugs. Herbal drugs are becoming more popularized and important even in the developed nations with hope of their non-toxicity and may play a role of substitute to overcome the problems of multi drug resistant pathogens. Cultivation of drug plants has been started in large scale without considering the land quality and in certain cases irrigation with waste water. Application of different fungicides, pesticides in that specific field or in neighboring fields may be directly deposited superficially or may be absorbed by the plant system. Therefore in the present situation possibilities of the Asian herbal drugs, contaminated with large number of toxic components cannot be ignored which may severely hurt human life in place of healing or curing. These contaminants may be either alone or in combination and may be originated intrinsically, extrinsically and deliberately. Certain contaminants may be highly toxic and may cause severe adverse effect in the human system. Some of the important contaminants are the heavy metals like lead, arsenic, cadmium, mercury, copper and pesticides. Adulteration of modern drugs to enhance drug potency and heterogenous microbial population including human pathogens may contaminate both raw and powdered herbal drugs. Among these contaminants, microbial contamination seems more severe and may be due to their dual action on the drug i.e. utilization of medicinally important chemical components as nutrients and in certain cases by producing certain mycotoxins, which may be even carcinogenic under certain conditions . Critical evaluation of both raw and powdered herbal drugs for contaminants is the urgent need of the time in order to provide safety measures in herbal health care medicine.
\end{abstract}

\section{Keywords: Contamination, Herbal drugs, Evaluation}

\section{INTRODUCTION}

Finding of healing potency in plants is an ancient idea. People in all continents have long applied poultices and imbibed infusion of hundreds if not thousands of indigenous plants dating back to pre history. Due to discovery and large application of antibiotics in 1950's plants have been ignored as a therapeutant in cure of human diseases. But currently medicinal plants are moving from fringe to mainstream use with great number of people seeking remedies and health approaches free from side effects caused by synthetic chemicals. During the last two decades the herbal-based drugs have become more popularized among developing countries and currently even in developed countries in management of human ailments.There are mainly three reasons behind their popularity, one is poverty among large sector of the society, secondly side effects of modern medicines mainly antibiotics and third is appearance of multidrug resistance of human pathogens. Currently much attention has been paid by scientists to find out substitutes and to utilize eco-friendly products for the prevention and cure of human diseases.
Albeit total number of plant species distributed in 16 agro climatic zones in India are 45000 but only 15000 have been recognized for their medicinal values as drug plants. Indian medicine system has identified only 1500 plants species for their medicinal values and hardly one third of this i.e. only 500 drug plants are being used in Ayurvedic pharmaceutical industries. There is a need to give more attention and to explore the therepeutic uses of the rest of drug plant species at national and international level. Besides this, maximum number of plants require their investigation as antimicrobial agents because an antibiotic has limited effective span. Therefore every year at least 2-3 antibiotics derived from micro organisms are launched (Clark, 1996).

Herbal drugs are crude preparations of different parts of one or more than one medicinal plant species. In other words, it can be stated that herbal drug is a dried medicinal plant or any part thereof, such as leaf, stem, root, flower or seed (Hitoshi et al., 1978). Large number of biologically active compounds from plant sources have shown their dramatic effect in modern medicine 
system. In the last few decades, some important plants origin chemicals compounds have been isolated and used in treatment e.g. 'quinine' for treatment of malaria, 'reserpine' for controlling hypertension, 'cocaine' as basis of modern anesthesia, and 'tubocurarine chloride' as muscle relaxant.

Earliest documented record of application of drug plants in the treatment of human diseases is available in 'Rigveda' 3500-1600 BC and 'Atharvaveda'. Ayurveda is based on ancient scripts written in Sanskrit Slokas by ancient 'Rishis' (Charak Samhita, Sushruta Samhita, Bhavaprakash Nighantu etc.). Ayurveda describes all aspects of human health management starting from diagnosis, treatment and each step of herbal drug formulation and preparation.

Albiet it is generally believed that herbal drugs have no adverse effect on human system but it is not scientifically true. Cowan (1999) reported the toxicity of plants to be used in various ways either as protectants or therapeutants. Adverse effect of herbal drugs may be due to their toxicity or due to some other reasons, which cannot be ignored. Since the last three years, large number of Chinese and Indian herbal drugs have captured the market and have also shown their negative results in certain cases. Under certain conditions these herbal drugs have been found to hurt human life in place of healing. Reports from patients experiencing negative health consequences caused by the use of herbal medicines are on the rise. Compositional guidelines must be compulsory in order to assure drug safety and minimize the adverse effects of herbal drugs. Compositional guideline is a list of tests, references to analytical and biological procedures and appropriate acceptance criteria which are numerical limits, range or other criteria for the tests described. One of the major causes of adverse effect is directly linked to the poor quality of herbal drugs. Various factors constitute to the poor quality of herbal medicines that can be discussed under the following headings :

\section{TOXIC CONTAMINANTS OF RAW AND POWDERED HERBAL DRUGS}

Both raw and powdered herbal drugs harbour wide variety of contaminants including heavy metals, pesticides, microbial pathogens and microbial saprophytes which seem to be a cause of deteriorating drug quality. The toxic contaminants may come from environment and conditions under which the medicinal plants are cultivated, grown and collected. Moreover, the conditions under which they are dried or processed including storage conditions and the conditions during transportation also contribute to the problem of toxic contamination. At certain sites industrial effluents are discharged containing heavy metals which may enter the plant system with absorption of water. These heavy metals may reach several plant parts with translocation.
Contamination due to cultivation of medicinal plant with chemicals and heavy metals from industrial effluents cannot be ignored.

1. Pesticides : Raw drug plant materials are liable to contain pesticide residues which accumulate from agricultural practices such as spraying for soil treatment and administration of fumigants during storage. Pesticides are also applied during the cultivation of herbs. Some wild herbs are not subjected to any pesticides. Some cultivated plants do not require the use of any pesticides because they have natural resistance to pathogenic organisms and insects. However some herbs are grown with pesticides. For example ginseng plant is highly susceptible to fungal rot, especially during its early growth period. Therefore fungicides are used during ginseng cultivation. However, care should be taken to avoid any chemicals close to the time of harvest. The natural processes (e.g. heavy rains) eliminate most of the fungicides prior to harvesting. The fungicide residues mainly quintozene have been reported in ginseng samples from Korea.

2. Fumigants : Fumigants are applied after the harvest of herbs. Sometimes large number of herbs are stored due to delay in their marketing. During these conditions, fumigants might be used in such a way to ensure that the entire supply is not affected by a rapidly multiplying insect pest. The use of fumigants are not desire d for herbs that are in high demand as they will not require long term storage. An example of fumigant used in the processing of herb is sulphur. Treatment with sulphur is mostly carried out on those herbs that are moist (e.g. ophiopogon) or those that discolour significantly over time (e.g. atractylodes). Several herbs may contain relatively higher levels of sulphur. This is mainly due to the processing method whereby herbs are spread on screens, underneath which is some heated sulfur. The sulphur fumes waft through the herb material and leave some residue. These sulphur residues are sometimes referred to as sulphites. However this method of preserving the herb quality is not known to cause reactions in sulphite sensitive individuals because sulphur is most prevalent element in human body and is essential to life.

3. Heavy metals : Contamination of raw herbal drugs and herbal products with heavy metals appeared in late 1990's, supplied from China and included extensive testing patent remedies by California health department, food and drug branch published in 1998. Both mercury and arsenic were found in a large number of samples. The presence of heavy metals in raw and finished herbal drugs may be due to natural contamination in the environment and also it may be intentional or unintentional contamination.

Unintentional contamination of herbal drugs is due to environmental contamination. Natural occurrence of heavy metals in the plants system is related with absorption of these heavy metals with translocation of 
water especially in areas where industrial effluent is being discharged and that contains huge amounts of these contents. Soil contaminated due to discharge of industrial effluents may be a major source for heavy metal contamination of raw herbal drugs and various aquatic reservoirs receiving industrial effluents being used for irrigation would also be playing important role in this context. The presence of heavy metals may also be the result of factory contamination during manufacturing process in the pharmaceuticals.

Sometimes heavy metals are added intentionally with a belief that these compounds may improve the effect of herbal drugs. Mercury and arsenic have been detected in several Chinese herbal products. The primary additives used are cinnabar that contains mercury and the additives on regular basis contain arsenic. Lead contamination of Chinese herbs may also occur due to intentional addition of lead compounds. Intentionally heavy metals are being recommended in the formulation of several Ayurvedic drugs with opinion of their importance in human health. However these metals are not used as such but in their oxidized forms and their doses are prescribed by specialized physicians.

The mechanism of the heavy metals associated with their toxic symptoms in the human body greatly varies and there is specific mechanism for specific heavy metal. Therefore to understand the mechanism of heavy metals in system needs a thorough and concentrated approach. Since heavy metals are highly toxic and their excess intake may cause severe damage in the different organs in the human system, therefore care should be taken for their use as alternative medicines.

4. Other contaminants in raw herbal drugs : Natural products may contain several natural contaminants. For example, plants naturally contain dead insects or insect parts. If the plant materials are not stored properly, the number of insects may be very high. Although insects are not considered harmful, they serve as an indicator of a handling. There may be a variety of other materials that fall in among the herbs such as paper, cloth twine etc, that may have been used during package of the herbs. 5. Toxic ingredients : The toxicity of the ingredients of herbal drugs is itself a complex factor due to intrinsic cause. Certain toxic components are being produced themselves as secondary metabolites and are stored in different parts of the plant system. Production of toxic components in the plant system is very specific and related to genetic nature of the plant and perhaps this is the only reason due to which certain plants become toxic or have toxicity as certain plants are known as poisonous plants.

6. Microbial contamination of herbal drugs : Medicinal plants serve as host and good niches for a wide spectrum of microorganisms with various individual properties and with considerable differences regarding qualitative and quantitative aspects. In principle the microbial load of plants is the result of a series of influences caused by animals and inanimate sources. The microbial contaminants are easily transferred by air and soil borne vectors as well as by irrigating water. The bacterial endospores and fungal spores are the two dominant groups of contaminants associated with medicinal plants. However, a broad diversity of bacterial, fungal cells can be found either in or in the plant material. Among these microorganisms, pathogens may also occur and this fact particularly limits the utilization of these plants, besides quality reduction caused by microbially induced spoilage.

Herbal medicines may be associated with a broad variety of microbial loading and exert an important impact on overall quality of herbal products and preparations. Generally the herbs are valued for their distinctive aroma, colour and flavour but unfortunately they are contaminated with high level of bacteria, moulds and yeasts and if untreated, the herbs can result in serious food borne illness (Aneesh et al., 2009). Factors that determine the microbiological quality of medicinal plants can be categorized into two types i.e. intrinsic and extrinsic (Kneifel et al., 2002). The intrinsic factors are nature of plant and natural barriers, structure of plant, plant composition (antimicrobial compounds and agents) and intracellular microbial contamination. while extrinsic factors include place of humidity, harvest method, post harvesting technological treatment, packaging and storage conditions and exogenous microbial contaminants. From a heterogenous group of microorganisms, association of several human pathogens specially under severely contaminated conditions could be due to handling by chronic carrier persons. Other sources may be good niches for their multiplication and may be the cause of certain diseases such as typhoid fever and certain viral diseases. Lenington (1967) reported the presence of Salmonella species from drug samples and dietary products. Erich et al. (2000) studied 138 medicinal herbal drugs and reported association of several microbial pathogens including enterohaemorrhagic E. coli, Staphylococcus aureus, Candida albicans and Campylo bacteria. They also found several fungi from these samples, which were potent mycotoxins producers. There are several related to production of mycotoxins by various fungal species from different countries. The presence of fungal species in crude herbal drugs and their role in spoilage of crude drug was reported by Matsushima et al. (1957). Hitoshi et al. (1978) reported three different kinds of mycotoxins i.e. aflatoxin, sterigmatoceistin and orchratoxin produced by different fungal genera from 14 powdered herbal drug samples. Aflatoxin seems to be very serious because of its association at upper limit with liver can cause cancer. Roy et al. (1988) demonstrated the production of 
aflotoxin B1 from $0.86-5.24 \mathrm{mg} / \mathrm{ml}$ in culture filtrate of $A$. flavous isolated from drug plants. Nagy et al. (1998) reported fungi and mycotoxin from 84 herbal drugs samples.

Storage conditions of raw herbal drugs in the developing countries are very poor and no efforts have been made to maintain proper conditions to minimize microbial population. Authors have recorded the presence of insects and even termites also. A cross contamination of these contaminants is also possible. Higher humidity facilitates enhancement of microbial population during storage of raw herbal drugs and causes severe loss of medicinally important chemical components which are utilized as nutrient components by harboured heterogeneous microbial flora.

Since raw herbal drugs harbour the microbial contaminants, therefore presence of these contaminants in powder herbal drugs appears very common. Their count varies with storage period and climatic conditions even after best storing packages and conditions. Prasad et al. (2002) made a quantitative enumeration of microbial flora of certain herbal drugs and found that all the drug samples were highly contaminated (Table 1). Albiet Hitoshi et al. (1978) found lesser number of fungi per gram sample from certain powdered herbal drugs including spices. Presence of lowest count of fungi reported by them needs reconfirmation of the data and storage condition etc. In certain drug samples least count may be related with toxicity of the ingredients of the samples. Esimone et al. (2007) evaluated seventeen solid and liquid preparation of herbal drugs for their microbial contamination. They found 74 bacterial and 55 fungal strains from these herbal preparation including Bacillus (35), Staphylococcus(11), Klebsiella (6), Listeria (5), Micrococcus (4), Cornybacterium(2), Proteus (2), Escherichia coli (2), Streptococcus (1), Acinetobacter (1), Citrobacter (1), Lactobacillus(1), and Serratia(1). Among the fungal isolates, Aspergillus, Penicillium, Candida and Mucor were miuch more common. This study showed a gross contamination of herbal drug preparation. Prasad et al. (2007) also reported the microbial contaminants of Triphala drug during storage period and their effect on drug efficacy against Salmonella typhi, Escherichia coli, Sarcina lutea, Bacillus megaterium, and Bacillus species (Table 2). Such type of reports are an evidence that medicines are much more prone for microbial attack and as such are the likely cause of being spoiled. The risk from these microbial contaminants may be extended in the form of certain health disorders if human pathogens are present there and are consumed as such.

Prasad et al. (2003) reported relationship between microbial flora and drug quality under laboratory conditions and found that ingredients of Triphla drugs lost their drug potency against certain pathogens, even $50 \%$ loss of drug potency was recorded within two months. It was concluded that herbal drug quality can be constantly maintained if storage conditions are up to the mark and proper treatment is being applied. These steps do not minimize the microbial count but modify the physical conditions that would yield a good result to protect the drug quality during storage period. Besides, this, the packaging and separation of raw herbal drugs will also be helpful in minimizing the cross contamination.

Smolinske (2005) reported an increase of herbal poisoning exposures by $344 \%$. He speculated that increased toxicity is related to various factors like lack of child -resistant packraging, new issues of contamination, proliferation of multiple ingredient products, excessive concentration of active ingredients and discovery of new drug - herb interactios.

7. Status of heavy metals in traditional Chinese medicines : A survey made in the USA from important Chinese herbal drugs showed that 24 products had 10 ppm lead, 36 products had $14.6 \mathrm{ppm}$ arsenic and 35 products contained an average of 1046 ppm mercury and 23 products had more than one contaminant. Besides this,

Table 1. Microbial load (cfu) per gram of certain powdered herbal drug during three months storage.

\begin{tabular}{lccccccc}
\hline \multicolumn{1}{c}{ Herbal drug } & $\begin{array}{c}\text { Dilution } \\
\text { factor }\end{array}$ & \multicolumn{2}{c}{ Microbial load at 3 } & Fongi & $\begin{array}{c}\text { rd } \\
\text { Actinom } \\
\text { ycetes }\end{array}$ & Bacteria & $\begin{array}{c}\text { Fungi } \\
\text { Actinom } \\
\text { ycetes }\end{array}$ \\
\hline Triphala churna & $10^{3}$ & $45 \times 10^{3}$ & $25 \times 10^{3}$ & - & $48 \times 10^{3}$ & $28 \times 10^{3}$ & - \\
Avippatikar & $10^{3}$ & $07 \times 10^{3}$ & $12 \times 10^{3}$ & - & $13 \times 10^{3}$ & $15 \times 10^{3}$ & $02 \times 10^{3}$ \\
Lavan Bhaskar & $10^{3}$ & $23 \times 10^{3}$ & $20 \times 10^{3}$ & - & $26 \times 10^{3}$ & $27 \times 10^{3}$ & - \\
Hingvashtak churna & $10^{3}$ & $102 \times 10^{3}$ & $13 \times 10^{3}$ & - & $111 \times 10^{3}$ & $28 \times 10^{3}$ & $01 \times 10^{3}$ \\
Aam ripu churna & $10^{3}$ & $05 \times 10^{3}$ & - & - & $11 \times 10^{3}$ & - & $02 \times 10^{3}$ \\
Payokil & $10^{3}$ & $04 \times 10^{3}$ & $05 \times 10^{3}$ & - & $08 \times 10^{3}$ & $08 \times 10^{3}$ & - \\
Control & $10^{3}$ & - & - & - & - & - & - \\
\hline
\end{tabular}

Source. Prasad et al. (2002) 
Table 2. Loss of drug potency of ingredients of Triphala drug at sixth month storage against certain bacterial test organisms.

\begin{tabular}{|c|c|c|c|c|}
\hline \multirow{2}{*}{ Test organisms } & \multirow{2}{*}{$\begin{array}{l}\text { Extract } \\
\text { concentration used }\end{array}$} & \multicolumn{3}{|c|}{ Percentage of drug potency loss at $6^{\text {th }}$ month } \\
\hline & & E. officinalis & T. bellerica & T. chebula \\
\hline Salmonella typhi & $100 \%$ & 45.0 & 62.5 & 45.3 \\
\hline Escherichia coli & $100 \%$ & 73.3 & 76.6 & 73.0 \\
\hline Sarcina lutea & $100 \%$ & 58.1 & 51.5 & 50.09 \\
\hline Bacillus megaterium & $100 \%$ & 75.0 & 77.7 & 66.0 \\
\hline Bacillus species. & $100 \%$ & 60.0 & 50.0 & 51.8 \\
\hline
\end{tabular}

Source. Prasad et al. (2003)

traditional Chinese medicines were also contaminated with Aristolachia causing severe health problems such intestinal nephropathy. Melchart et al. (2001) analysed 317 batches of dried Chinese medicinal herbs supplied to German Hospitals which were certified free from contaminants. They reported that $3.5 \%$ of samples were contaminated with double concentration of several heavy metals which were beyond the permissible limit recommended by WHO that included lead, mercury, cadmium etc. Koh and Woo (2000) reported that Chinese herbal medicines were contaminated with different heavy metals and in certain traditional Chinese medicines, mercury seems a part of preparation such as Cinnabaris (mercury sulphide). Wong et al. (1993) reported the presence of cadmium, cobalt, copper, iron, manganese, nickel, lead, zinc and mercury from 42 Chinese herbal samples which were analyzed spectrophotometrically. 8. Status of heavy metals in Indian medicine system : Large number of drugs of Indian medicine systems are prepared by using heavy metals such as lead, iron, gold, mercury, silver, zinc etc. The Indian physicians recognized the toxicity of heavy metals and accordingly they had given the directions for various physical and chemical processes of drugs for their detoxification and recommended their application for maintaining human health.

There are reports available about the presence of heavy metals in Indian herbal drugs also. Itankar et al. (2001) made a survey of 31 Indian Ayurvedic formulations and only one drug was reported to contain $1 \mathrm{ppm}$ and 16 formulations contained double the quantity of heavy metals than that of recommended limit by WHO. Lalla et al. (2004) made atomic absorption and spectrophotometric studies of the powdered fruits of Amla, Baheda, Harad and marketed samples of Triphala along with laboratory prepared Triphala and observed the presence of lead. However, it was within the limit recommended by WHO. A large number of cases of led poisoning associated with Indian medicines have been reported since last three decades. Robert et al. (2004) collected Asian herbal drugs from supermarkets, markets and various stores; and they found the presence of detectable amount of lead, mercury and arsenic at ratio of $20 \%$ of the samples tested. They concluded that drug samples contained potentially harmful level of these metals and that may cause metal toxicity in the users.

Robert et al. (2008) made a survey of safes of Indian medicines through internet and found 25 websites featuring 673 medicines. They could procure 193 only. Out of these 193 Ayurvedic medicines, 115 were U.S. manufactured and 77 were of Indian make. Among these, Rasa shatra medicines were 32 in number and rest were plants based. About $20 \%$ drugs contained detactable amount of either lead, mercury and arsenic alone or in combined form. Recently, a study was conducted by Harvard Medical School on Indian herbal drugs and found potentially harmful level of lead, mercury and arsenic in Bal Ghutti, Maha Yograj Guggul, Mahalaxmi Vilas Ras, Safi and Shilajit etc. ( Aneesh et al., 2009). 9. Status of microbial contaminants of herbal drugs : Albeit raw and powdered herbal drugs contain a heterogeneous group of microbial count but efforts have been made to investigate either fungal contamination and spoilage of crude drugs (Matsushima et al., 1957 and 1958). Fungal count enumeration and mycotoxin production were carried out by some workers. Important contributions are that of Hitoshi et al., 1978; Nagi Halim Azij et al., 1998; Roy and Chaurasia, 1998. Fragmentary reports on microbial contaminants of raw and powdered herbal drugs provide the picture of herbal drugs quality as well as their possible effect on health management. Studies in all the cases are limited only to observe mycotoxin production. Ray (2003) has given a vast information of fungal contamination of herbal drugs. In most of the cases, herbal drugs were found contaminated with fungi and showed potent mycotoxin producers. However, the degree of mycotoxin production was variable among different drug plants which may be related with substrates present in different drug plants. It may also be related with genetic nature of plants. Wong wiwat et al. (2004) studied 20 herbal drug samples of Thailand and revealed that $18 \%$ of herbal samples were contaminated with detectable amount of aflatoxins ranging 
from 1.7 to $14.3 \mathrm{mg} / \mathrm{g}$. It may possible that the original fungal infection of these products may have been derived from either the crude herbal or other ingredients making these preparations,such as starch. Ernst (2005) studied the microbial contamination of two Indian herbal drugs and found that both drugs were contaminated with Bacillus subtilis, B.coagulans and B.cereus; and with four fungal genera.

It is obvious that all the herbal drug samples worldwide are harbouring microbial population but their quantity and quality vary. Prasad et al. (2002) made a quantity enumeration of certain herbal drugs used in treatment of several diseases. Ignored aspects of microbial contaminants of herbal drugs need more attention on the influence of these on drug potency, their application and enhancement of therapeutic spectrum of drugs. A survey of literature indicates that microbial flora of both raw and powdered herbal drugs is only limited to microbiological load, in certain cases to mycotoxin analysis and human pathogens. The important aspect that effect of these microorganisms on drug potency in relation of chemical changes is an urgent need of the time. Besides thus, efforts should also be made to utilize the habitated microbial flora of herbal drugs to enhance the threpeutic spectrum by determining their antagonastic nature against human pathogens.

\section{Major effects of microbial contaminants in human health} management program:

Since various medicinally important components are being utilized by heterogenous groups of adherent microbial flora during storage, the phytochemicals of the raw and powdered herbal drugs are drastically changed both quantitatively and qualitatively during that period. Hence significance of phytochemical analysis of the specific plants and their parts becomes insignificant unless storage conditions are being optimized to keep the level of the phytochemicals constants. Doses prescribed in indigenous medicine system in powdered herbal drug are effected due to loss of ingredients and therefore both loading and symptomatic relief may be varied.

Certain microbial contaminants may be serious human pathogens.Mycotoxins produced by certain fungal genera may cause severe health hazard. Several complications and disorders in certain humans may occur due to ingestion of adherent fungal flora with herbal powdered drugs. It is indicated that initially in the lower gut of human body there are about $4-8$ lbs of friendly bacteria. This comprises about $85 \%$ of all organisms in the bowels. The remaining $15 \%$ is fungus. It takes the fungus incheck However, its excess is toxic and much more powerful then bcteria. Fungi have no antibodies to protect itself but produce deadly mycotoxins that may result in failure of digestion and assimilation of all the nutrients. Organs and glands are deprived of its building blocks and systems begin to fail. In order to lower down the risk with adherent microflora of powdered herbal drugs, precaution should be taken that drugs should be properly sterilized befor their intake.

\section{Conclusion and recommendations for future strategies}

1. More research is needed to evaluate herbal drugs both raw and finished products for their contaminants. No raw and powdered herbal drug should be approved at any cost containing the concentrations of those specific heavy metals above the concentration recommended by WHO.

2. Examination of microbial flora must be evaluated and efforts should be made to identify the genera and species in order to prevent out break of disease and food poisoning.

3. India pharmacopia (Ayurvedic) is silent regarding microbial flora. Although in Chinese herbal drugs provisions have been given but are only limited upto the reports of their count (CFU) which do not appear to be correct and need reconfirmation.

4. Entire study of microbial contamination of herbal drugs is centralized with CFU. Analysis of toxins and more research is needed to find out various factors in storehouses to minimize their count.

5. Emphasis on microbial population and loss of medically important chemical components utilized by these organisms under different conditions should be evaluated in order to maintain a constant nature of the drugs. 6. Optimization of the various factors affecting multiplication of microbial flora should be found out. Based on findings,optimum conditions should be maintained in the store-houses to minimize microbial population in order to maintain drug quality.

7. Special attention should be drawn in storage of raw herbal drugs to prevent the cross contamination of one to other raw drug material. Therefore separate chambers should be provided for each raw drug. Moreover, the raw drugs can be separately packed in the pre sterilized polythene bags/packets before storage.

8. Before processing, raw herbal drugs may be treated with gama irradiation in the range of 3-10 KGy to reduce the total aerobic viable cell counts in highly contaminated spices and drug herbs.

9. Database information regarding analysis for the parameters which are responsible for deterioration of the quality of drugs in one or other way should be maintained for herbal raw and finished products/complementary medicines/therapeutic goods by the Central govt. of India. 10. The therapeutic spectrum of the herbal drugs and drug potency should be enhanced by using the knowledge of traditional medicine system and modern medicine system. This may provide not only a wide range of application but also a suitable base for drug resistant 
pathogens.

11. Herbal medicines may be associated with low or high risk depending on the toxicity of their ingredients, proposed dose, management and the potential for adverse reactions if any. Therefore all herbal medicines should be evaluated for safety, quality and efficacy on the Australian pattern and then registered . Therefore, the standard operating procedures, good laboratory practices, goods supply practices and goods manufacturing practices must be followed besides the advanced chemical and biomedical researches.

\section{REFERENCES}

Aneesh, T.P., Mohamed Hishan, M. Sonal Shekhar, Manju shree Madhu and T.V. Deepa (2009). International market scenario of traditional Indian herbal drugs- India declining. International. J. of Green Pharmacy, 184-190.

Clark, A.M. (1996). Natural products as a resource fro new drug. Pharma research 13: 1996.

Cowan M.M. (1999). Plant product as antimicrobial agent. Clinical Microbiology Review, 564-582.

Erich Czech, Wolfgang Kneifel and Brigitte Kopp. (2000). Microbiological status of commercially available medicinal herbal drugs- A screening study. Plant Medica, 67, 262-269.

Ernst, E. (2005). Contamination of herbal medicines. The Pharmaceutical Journal, 275: 167-168.

Esimone, C.O., P.O. Olighe, U.E. Dibaa, and E.C. Ibezium. (2007). Gross microbial contamination of herbal medicinal products (HMPs) marketed in mid western Nigeria. International Jour. of Molecular Medicine and Advace Sciences, 3(2): 87-92.

Hitoshi Hitokoto, Satoshi Marozumi, Tomoaki Ki Wauke, Sanzo Sakai and Hiroshi Kurata. (1978). Fungal contamination and mycotoxin detection of powdered herbal drugs. Applied and Environmental Microbiology, 36 (2): 252-256.

Itankar P.R., P.R. Sakharkar, A.V. Chandran and A.T. Patel (2001). Estimation of arsenic contents in some Ayurvedic formulations. Hamdard Medicus, 19: 95107.

Koh H.L. and S.O. Woo (2000). Chinese proprietary in Singapore. Regulatory control of toxic heavy metals and undeclared drugs. Drug Safety, 23: 351-362.

Kneifel wolfgang, Erich Czech and Kopp Priegithe. (2002). Microbiological status of commercially available medicinal herbal drugs. A screening study. Planta Medica, 67: 263-269.

Lalla, J.K., P.D. Hamrapurkar and H.M. Mamania. (2004). Mineral content and microbial impurity of Triphala Churna and its raw material. Indian J. of Traditional Knowledge, 3(1): 86-91.

Lennington, K.R. (1967). Salmonella in drugs and dietary supplements. Drugs Cosmet. Ind., 100: 42-43.
Matsushima, T., H. Itoh and M. Ikeda (1957). Investigation on the fungal spoilage of crude drugs I. J. Japan Bot., 32: 9-15.

Matsushima T., H. Itoh and M. Ikeda (1958). Investigation on the fungal spoilage of crude drugs II. J. Japan Bot., 33: 12-23

Melchart, D., H. Wagner, Hager, S., Saller, R. and Earnst, E. (2001). Quality assurance and evaluation of Chinese medicine drug in a hospital of traditional Chinese medicines in Germany: a five year report. Alternative Therapies, 7:524.

Nagy Halim Azij, Yousuf A., Yousuf, Moheiz, E. L. foully and Lotfy A. Moussa (1998). Contamiantion of some common medicinal plant samples by fungi and their mycotoxins. Bot. Bull. Acad. Sin., 39: 279-285.

Prasad, G, Sharma, N. and Gupta, K. K. (2002). Quantitative enumeration of microflora of certain herbal Ayurvedic drugs during storage. Hamdard Medicus (1): 73-77.

Prasad G., Metiyan, P., Gupta, K. K., Sharma, P. and Prashant (2003). Microbial flora and drug efficacy related with enhancement of therapeutic spectrum of three ingredients of Triphala drug during storage. Hamdard Medicus. XLVI (4): 82-87.

Prasad, G., Sharma, P., Gupta, K.K. and Chopra, A.K. (2007). Effects of microbial contaminants on Triphala drug efficacy during storage and enhancement of therapeutic spectrum of the drug in vitro. Hamdard Medicus, 50 (3): 55-62.

Robert, B., Saper, Stefanos, N., Kales, Janet Paquin, Michael J., Burns, David M., Eisenberg, Roger B., Davis, and Russell S., Phillips. (2004). Heavy Metal Content of Ayurvedic Herbal Medicine Products. JAMA, 292(23): 2868-2873.

Robert B., Saper, Russel S., Phillips and Sehgal, Amisha (2008). Lead, Mercury and Arsenic in U.S. and Indian manufactured Ayurvedic Medicines sold via the internet, JAMA, 300 (8): 915-923.

Roy, A.K. and Chaurasia, H.K. (1988). Aflatoxin contamination of some common drug plants. Appl. Environ. Microbiol. 54: 842-843.

Roy, A.K. (2003). Mycological problems of crude herbal drugs overview and challenges. Indian Phytoph., 56 (1): $1-13$.

Smolinske,C.Susan (2005). Herbal product contamination and toxicity. Journal of Pharmacy Practice, 18 (3): 188208

Wong, M.K., Tan, P. and Wee, Y.C. (1993). Heavy metals in some Chinese herbal plants. Biol. Trace Elements Res., 36 (2): 135-142.

Wong wiwat, Tassaneeyakul, Ebrahim Razzazi-Fazeil, Supatra Porasuphathana and Bohm Josef (2004). Contamination of aflatoxins in herbal medicinal products of Thailand. Mycopathologia 158: 239-244. 